\title{
Sesquiterpene alcohol cedrol chemosensitizes human cancer cells by destabilizing plasma membrane lipid rafts and inhibits cell proliferation
}

\author{
Siddhartha Mishra ${ }^{1}$, Yun Soo Bae ${ }^{2}$, Yong-Moon Lee ${ }^{3}$, Jae-Sung Kim ${ }^{4}$, Seung-Hyun $\mathrm{Oh}^{5}$, \\ and Hwan-Mook Kim ${ }^{5}$ \\ ${ }^{1}$ Dr Hari Singh Gour University \\ ${ }^{2}$ Ewha Womans University \\ ${ }^{3}$ Chungbuk National University \\ ${ }^{4}$ University of Florida \\ ${ }^{5}$ Gachon University of Medicine and Science
}

May 11, 2020

\begin{abstract}
Background and Purpose: Chemosensitization of cells with small molecules may improve the therapeutic index of antitumoral agents by making tumor cells sensitive to the drug regiment and thus overcoming treatment resistance and side-effects of single therapy. Cell membrane lipid rafts are known to transduce various signaling events in cell proliferation. Sensitizing cancer cells may cause modulation of membrane lipid rafts which may potentially be used in improving anticancer drug response. Experimental Approach: Cedrol, a natural sesquiterpene alcohol, was used to treat human leukemia K562 and colon cancer HT-29 cell lines and effects were observed. Key Results: Cedrol decreased the cell viability by inducing apoptosis in both cell lines by activation of pro-apoptosis protein BID and inhibition of anti-apoptosis proteins Bcl-XL, Bcl-2, and XIAP. Cedrol activated caspase-9 dependent mitochondrial intrinsic pathway of apoptosis. Furthermore, cedrol inhibited the levels of pAKT, pERK and pmTOR proteins as well as nuclear and cytoplasmic levels of p65 subunit of NF- $x$ B. Cedrol caused redistribution of cholesterol and sphingomyelin contents from membrane lipid raft, which was confirmed by synergistic inhibition in combination with methyl$\beta$-cyclodextrin (lipid raft disrupting agent). Lipid raft destabilization by cedrol led to the increased production of ceramides and inhibition of membrane bound NADPH oxidase 2 enzyme activity. Conclusions and Implications: Cholesterol/sphingomyelin redistributing abilities of cedrol appear as novel mechanism of growth inhibition of cancer cells. Cedrol can be classified as a natural lipid raft disrupting agent with possibilities to be use in general studies involving membrane lipid raft modifications.
\end{abstract}

\section{Hosted file}

BJP Cedrol MS.doc available at https://authorea.com/users/320425/articles/449992sesquiterpene-alcohol-cedrol-chemosensitizes-human-cancer-cells-by-destabilizing-plasmamembrane-lipid-rafts-and-inhibits-cell-proliferation 
(A)

(C)

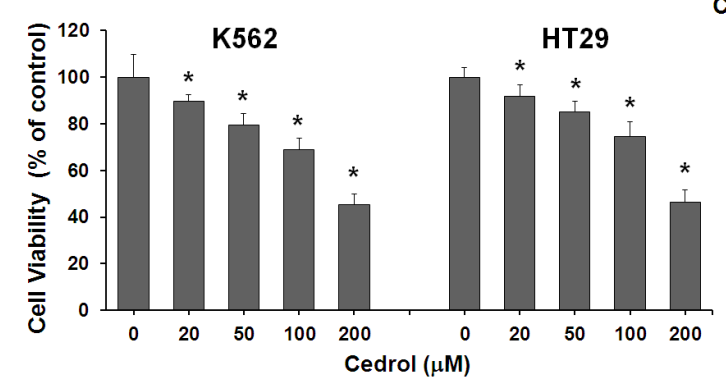

Cedrol $(\mu \mathrm{M}) \quad 0 \quad 100 \quad 200$

(B)
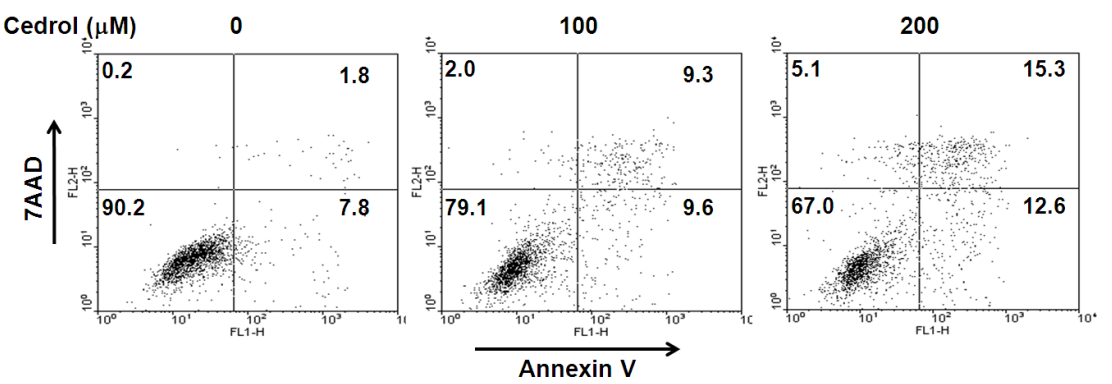
(A)

$$
\begin{aligned}
& \text { z-VAD-fmk } \\
& \text { Cedrol }(\mu \mathrm{M})
\end{aligned}
$$

Pro-Caspase-3

\section{GAPDH}

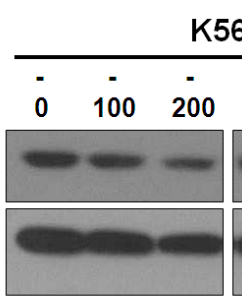

K562

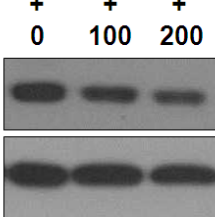

(B)

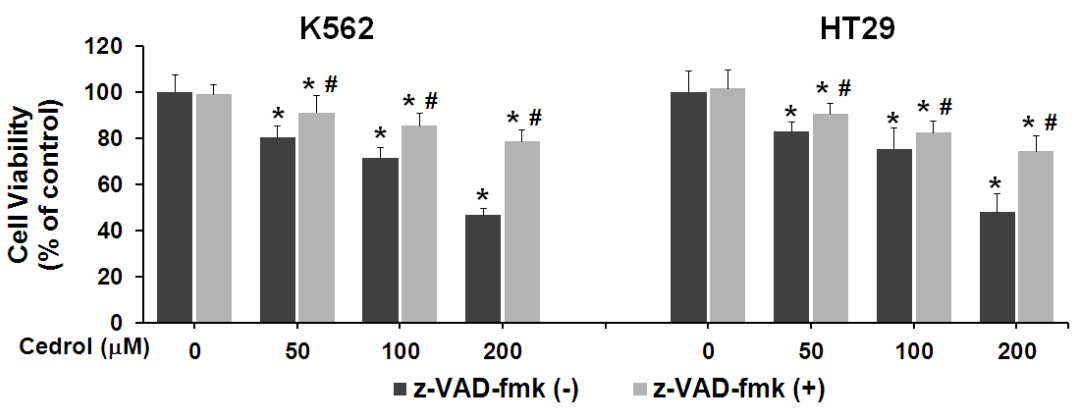

(C)

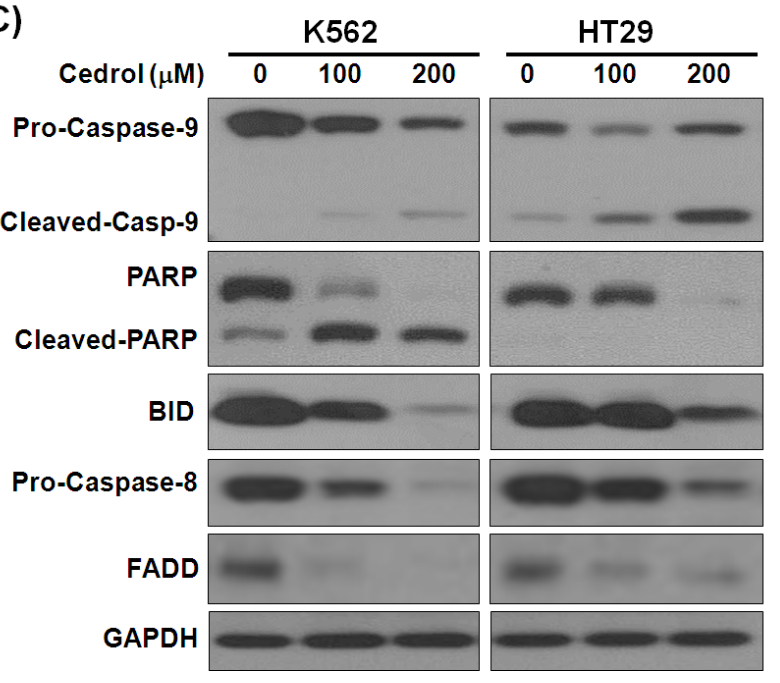

HT29

\begin{tabular}{cccccc}
\hline- & - & - & + & + & + \\
0 & 100 & 200 & 0 & 100 & 200
\end{tabular}

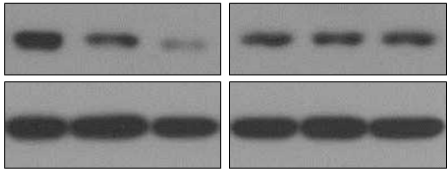

HT29 


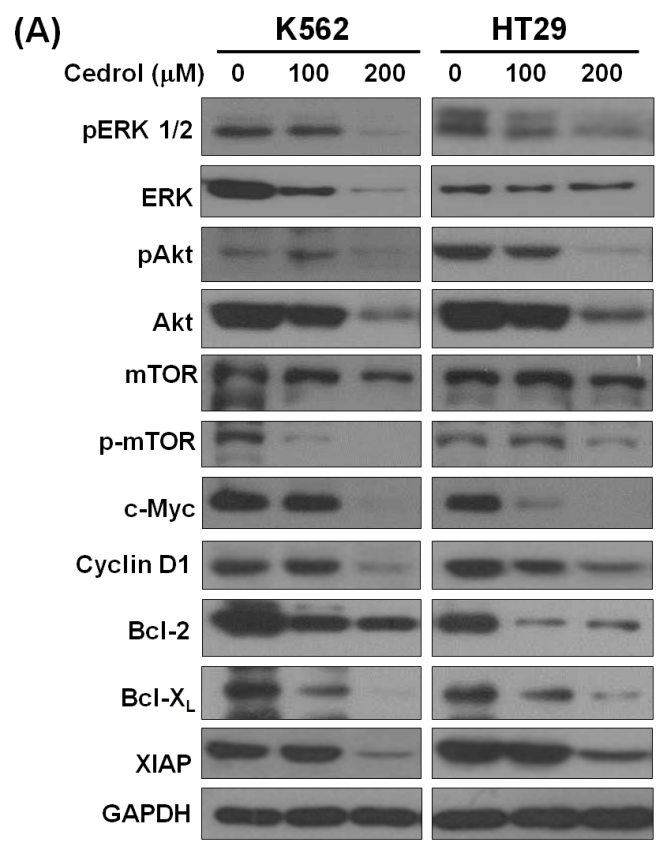

(B)

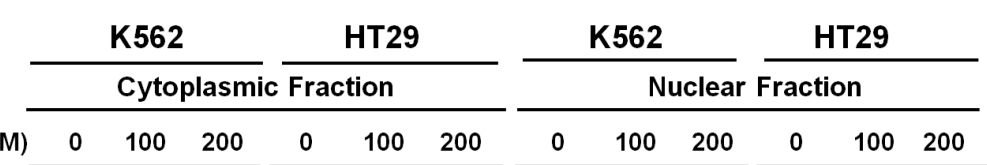

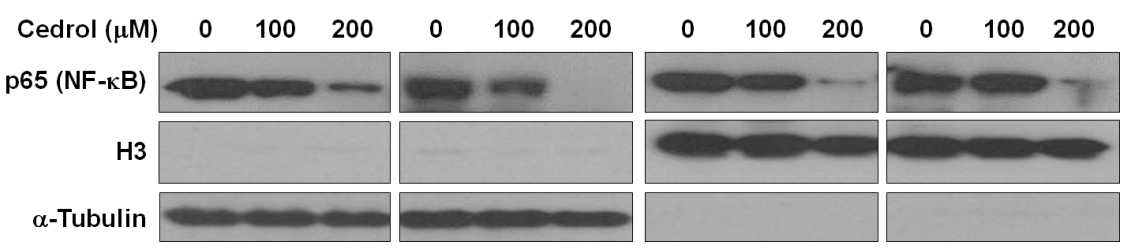


(A)

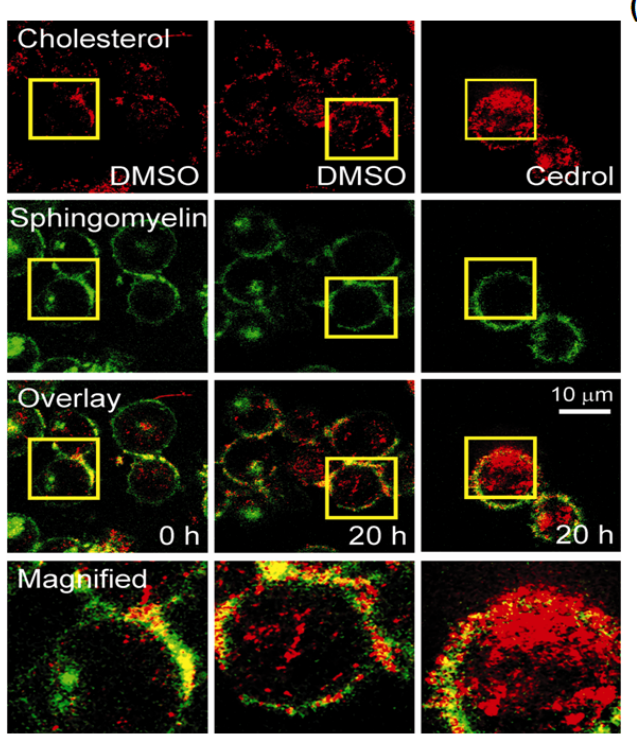

(B)

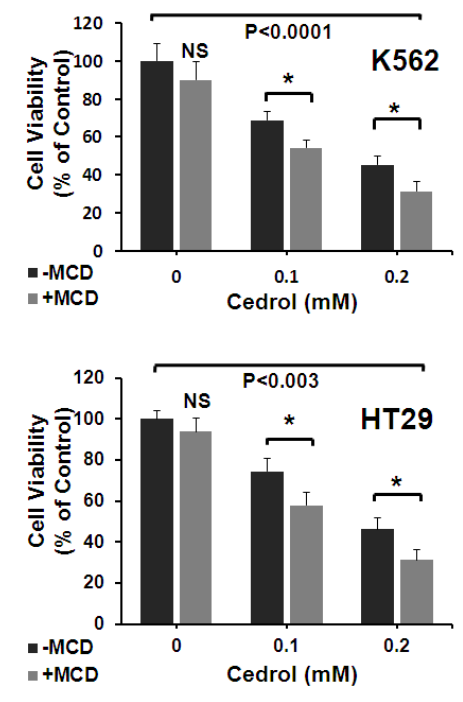

(C)

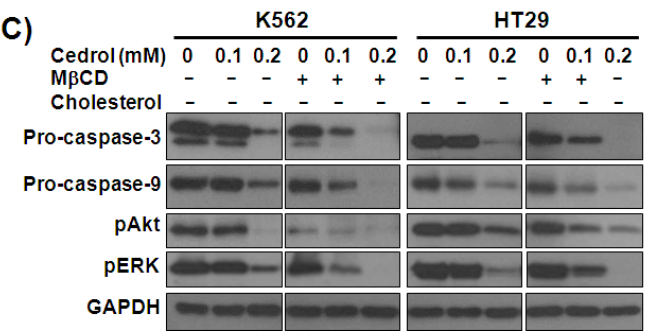

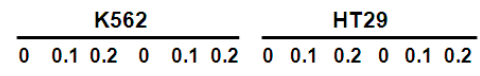

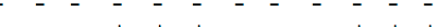
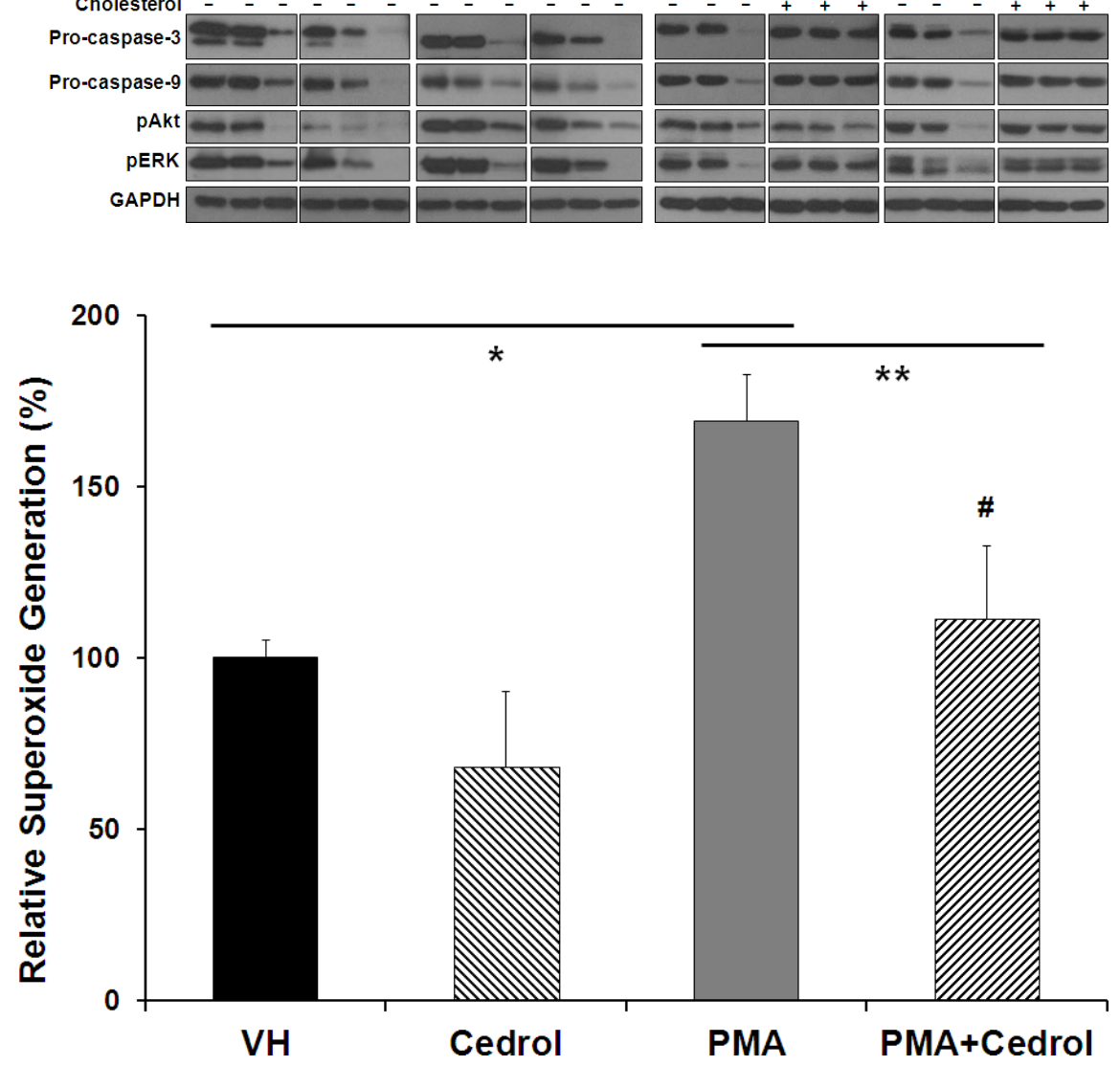


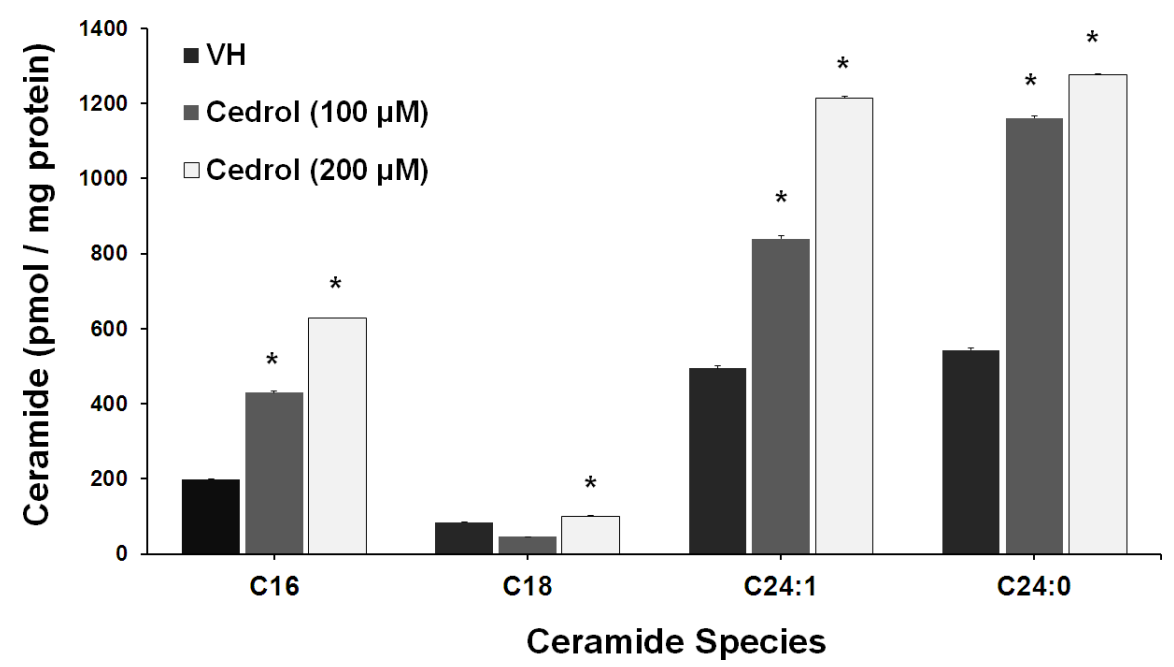

